\title{
AAALAC International Standards and Accreditation Process
}

\author{
Montip Gettayacamin ${ }^{1}$ and Leslie Retnam ${ }^{2}$ \\ ${ }^{1}$ AAALAC International, 5205 Chairman's Court, Suite 300, Frederick, MD, USA \\ ${ }^{2}$ Veterinary Services, Biological Resource Centre, $A^{*} S T A R$, Singapore
}

\begin{abstract}
AAALAC International is a private, nonprofit organization that promotes humane treatment of animals in science through a voluntary international accreditation program. AAALAC International accreditation is recognized around the world as a symbol of high quality animal care and use for research, teaching and testing, as well as promoting animal welfare. Animals owned by the institution that are used for research, teaching and testing are included as part of an accredited program. More than 990 animal care and use institutions in 42 countries around the world (more than 170 programs in 13 countries in the Pacific Rim region) have earned AAALAC International accreditation. The AAALAC International Council on Accreditation evaluates overall performance and all aspects of an animal care and use program, involving an in-depth, multilayered, confidential peer-review process. The evaluators (site visitors) consider compliance with applicable local animal legislation of the host country, institutional policies, and employ a customized approach for evaluating overall program performance using a series of primary standards that include the Guide for the Care and Use of Laboratory Animals, the Guide for the Care and Use of Agricultural Animals in Research and Teaching, or the European Convention for the Protection of Vertebrate Animals Used for Experimental and Other Purposes, Council of Europe (ETS 123), and supplemental Reference Resources, as applicable.
\end{abstract}

Key words: Accreditation, International, AAALAC

\section{INTRODUCTION}

Short history. AAALAC International promotes the responsible treatment of animals in science through a voluntary accreditation program. AAALAC International is a non-governmental, non-profit organization. The accreditation program was established in 1965, when leading veterinarians and researchers organized the American Association for Accreditation of Laboratory Animal Care (AAALAC), as a private, nonprofit organization. In the years that followed, AAALAC accredited hundreds of organizations across the United States, raising the benchmark for laboratory animal care to new heights. In 1996, AAALAC changed

Correspondence to: Montip Gettayacamin, AAALAC International, 5205 Chairman's Court, Suite 300, Frederick, MD, USA 21703 E-mail: montipg@aaalac.org

This is an Open-Access article distributed under the terms of the Creative Commons Attribution Non-Commercial License (http:// creativecommons.org/licenses/by-nc/3.0) which permits unrestricted non-commercial use, distribution, and reproduction in any medium, provided the original work is properly cited. its name to the Association for Assessment and Accreditation of Laboratory Animal Care International (AAALAC International). The name change reflected the organization's recognition in other countries, and its commitment to enhancing life sciences and quality animal care around the world. In 2016 AAALAC eliminated spelling out the acronym in favor of simply using "AAALAC International" as its legal, official name. As AAALAC International's accreditation program has grown to encompass many wildlife and agricultural animal research programs, removing the use of the word "laboratory" previously used in conjunction with the AAALAC acronym allowed for greater inclusivity. Participating institutions receive an independent, unbiased expert assessment, and those that meet or exceed applicable standards are awarded accreditation (1).

Accreditation around the Globe. AAALAC International is the only organization that accredits animal care and use programs for research, teaching and testing around the globe. Any institution using animals for these purposes is eligible to apply for AAALAC accreditation. This includes universities, pharmaceutical and biotechnology companies, 
agricultural research programs, hospitals, government agencies and nonprofit organizations. The appropriate administrative entity making application is the accreditable program and follows the Rules of Accreditation (https:/www.aaalac.org/accreditation/rules.cfm/), and its animal care and use programs and facilities are evaluated to determine accreditation status. All animal programs and facilities of the accreditable institution must be included in the application materials and evaluated (1).

For more than 52 years, the scientific community has actively - and voluntarily - participated in AAALAC International's accreditation program. Today, more than 990 organizations worldwide in 42 countries are accredited by AAALAC International (as of February 2017). Approximately 97\% of these institutions maintain a full accreditation status, with approximately $3 \%$ in less than full accreditation status, which includes full accreditation with condition(s), deferred accreditation or probationary accreditation. Institutions in a less than full accreditation status are actively working on correcting one or more mandatory deficiencies.

Accreditation in the Pacific Rim region. There has been a marked increase in the number of institutions seeking/attaining accreditation in the Pacific Rim region. Currently there are 177 accredited programs $(>17 \%$ of total accredited programs globally) located in the Pacific Rim region. The People's Republic of China has the largest number of accredited programs in this region (68 programs or $38.4 \%$ of total), followed by 27 accredited institutions in Japan (15.3\%). There are 22 units in India $(12.4 \%) ; 21$ units in Korea $(11.9 \%) ; 16$ units in Taiwan Republic of China (9\%); six units each in Thailand and Singapore (3.4\% each); two units each in Australia, Indonesia and Malaysia (1.1\% each); and one unit each in Philippines and Vietnam ( $0.6 \%$ each). The demographics of institutions accredited in the Pacific Rim region (Table 1) show that $67.2 \%$ (119 institutions) are commercial institutions (e.g., contract research organizations, pharmaceutical companies, animal producers, etc.); 24 institutions $(13.5 \%)$ are academic institutions. Three are campus-wide accredited programs and 19 are university limited programs in which only some of the schools, faculties, centers or institutions of the universities are accredited; 14 institutions $(8 \%)$ are non-profit organizations; 13 units $(7.3 \%)$ are governmental institutions; and seven are hospitals (4\%).

\section{ACCREDITATION PROGRAM}

Council on accreditation. AAALAC International's Council on Accreditation (CoA) is a group of volunteer experts responsible for evaluating animal care and use programs, with the ultimate responsibility for determining accreditation status. Members of the CoA are chosen and appointed to serve based on their extensive experience in the fields of veterinary medicine, laboratory animal science or animal research, and their commitment to humane animal care and use (1). The CoA is currently comprised of more than 60 qualified and experienced animal care and use professionals and researchers from around the globe. They conduct the site visits to evaluate programs and determine which institutions are awarded AAALAC International accreditation. The Council includes North American, European and Pacific Rim sections which evaluate programs in their respective regions.

AAALAC international standards. AAALAC International is not a regulatory body and does not develop regulations. AAALAC International relies on Three Primary

Table 1. Type and number of acredited institutions in the thirteen Pacific Rim countries

\begin{tabular}{|c|c|c|c|c|c|c|c|c|c|}
\hline \multirow{2}{*}{ Countries } & \multirow{2}{*}{$\begin{array}{c}\text { Total \# of } \\
\text { accredited units }\end{array}$} & \multicolumn{4}{|c|}{ Commercial } & \multirow{2}{*}{ Academia } & \multirow{2}{*}{ Non-profit } & \multirow{2}{*}{ Government } & \multirow{2}{*}{ Hospital } \\
\hline & & CRO & Pharma & Animal producer & Other & & & & \\
\hline P. R. China & 68 & 26 & 12 & 11 & & 8 & 4 & 6 & 1 \\
\hline Japan & 27 & 9 & 13 & 2 & & 2 & 1 & & \\
\hline India & 22 & 12 & 4 & 4 & & & 2 & & \\
\hline Republic of Korea & 21 & 3 & 2 & 4 & & 5 & 1 & 4 & 2 \\
\hline Taiwan R.O.C. & 16 & 3 & 1 & 2 & & 2 & 4 & 1 & 3 \\
\hline Singapore & 6 & 1 & & 1 & 1 & 1 & 1 & & 1 \\
\hline Thailand & 6 & & & & & 3 & 1 & 2 & \\
\hline Cambodia & 3 & & & 3 & & & & & \\
\hline Australia & 2 & 1 & & & & 1 & & & \\
\hline Indonesia & 2 & & 1 & & & 1 & & & \\
\hline Malaysia & 2 & 1 & & & & 1 & & & \\
\hline Philippines & 1 & & & 1 & & & & & \\
\hline Vietnam & 1 & & & 1 & & & & & \\
\hline Total number & 177 & & & 119 & & 24 & 14 & 13 & 7 \\
\hline$\%$ of Total & 100 & & & 67.2 & & 13.5 & 8.0 & 7.3 & 4.0 \\
\hline
\end{tabular}


Standards for evaluating laboratory animal care and use programs: the Guide for the Care and Use of Laboratory Animals (Guide) (2) the Guide for the Care and Use of Agricultural Animals in Research and Teaching (Ag Guide) (3) and the European Convention for the Protection of Vertebrate Animals Used for Experimental and Other Scientific Purposes (ETS 123) (4) along with other widely accepted guidelines.

In addition, AAALAC International does publish "Position Statements", http://www.aaalac.org/accreditation/positionstatements.cfm/ that can be used as supplemental guidance when dealing with certain issues, such as the definition of laboratory animals; the role of the Attending Veterinarian and veterinary care; cage or pen space standards; social housing requirements; selecting the appropriate standard(s) for the care and use of agricultural animals; safety requirements for walk-in cage/rack washers and bulk sterilizers; the occupational health and safety program; and safety precautions for Macacine herpesvirus 1 (1).

Since 1975, AAALAC International has also referred to other peer reviewed specialty publications for supplemental information about procedures or techniques related to the care and use of laboratory animals. These publications are designated as Reference Resources (http://www.aaalac.org/ accreditation/resources.cfm/). These references cover many topics including both general and specific areas of interest. Specific areas of interest include; biosafety; education; euthanasia; health monitoring; occupational health and safety; re- search; and information on specific species of animals. All references included on the list have been formally reviewed and adopted by the CoA as guidance for accredited units as well as for the use by AAALAC International representatives during the site visit (1). For example, acceptable euthanasia methods are established by guidance provided in the AVMA Guidelines for Euthanasia of Animals: 2013 Edition (5), and occupational health and safety issues are evaluated against the standards found in the book, Occupational Health and Safety in the Care and Use of Research Animals (6), in addition the standards of the Guide, which are always applied. Also, these references may be utilized during Council deliberations when discussing issues identified during site visits. When applicable, clarifying notes are appended to the reference, to provide additional information on any exclusions or clarification in the reference occurring as a result of the review by the CoA (1).

Since the release of the Guide, twenty-seven Frequently Asked Questions (FAQs) have been developed, and are continually revised as needed to include new information, http://www.aaalac.org/accreditation/faq_landing.cfm/. Two of the most recent FAQs provide clarification on the use of carbon dioxide $\left(\mathrm{CO}_{2}\right)$ for euthanasia, and the transportation of animals used in research.

AAALAC international ad hoc consultants/specialists. AAALAC International maintains a worldwide pool of more than 300 ad hoc Consultants/Specialists who have expertise beyond the realm of traditional laboratory animal species as well as specific expertise (e.g., aquatics, agricultural science). Many also have competencies in unique disciplines, such as applied neuroscience, behavioral science, toxicology, pharmacology or physiology. Ad hoc Consultants/Specialists accompany Council members on site visits and provide input during the evaluation process. These specialists add significant depth, knowledge and experience to the site visit team (1).

The Guide for the Care and Use of Laboratory Animals. The Guide (2) is the primary standard commonly used for most institutions in the Pacific Rim region seeking and attaining AAALAC International accreditation. The Guide has been translated to several languages including Mandarin Chinese, Japanese, Thai, Traditional Chinese, Portuguese, and Malaysian. The goal of the Guide is to promote the humane care and use of laboratory animals by providing information that will enhance animal well-being, the quality of research, and the advancement of scientific knowledge that is relevant to both human and animals (2). The Guide is organized into five chapters. Chapter 1 describes key concepts for the humane use of animals used in research, testing or teaching, and the following four Chapters provide recommendations for standards for animal care and use programs; environmental, housing and management; veterinary care and physical plant, respectively.

Engineering, performance and practice standards are key terms used in the Guide (2).

- Engineering standards are a standard or guideline that specifies in detail a method or technique for achieving a desired outcome; it does not provide for modification in the event that acceptable alternative methods are available or unusual circumstances arise. Engineering standards are prescriptive and provide limited flexibility for implementation (2).

Examples of engineering standards in the Guide are the recommended macroenvironmental temperatures for common laboratory animals, such as; maintaining relative humidity between $30 \%$ to $70 \%$; provision of $10-15$ fresh air changes per hour in animal housing rooms; maintaining light-levels between 130 and 325 lux in the room at cage level; ensuring noise levels are less than $85 \mathrm{~dB}$; minimum cage space requirements for commonly used laboratory animals; animal food stored at temperatures between $<21^{\circ} \mathrm{C}$ and $<50 \%$ relative humidity ; sanitation frequency of solidbottom caging, bottles, sipper tubes sanitation at least once a week, and enclosures and cage tops sanitation at least once every two weeks; minimum temperature requirement for rinse water at $82.2^{\circ} \mathrm{C}\left(180^{\circ} \mathrm{F}\right)$; heating, ventilation and air conditioning capable of adjustments at $\pm 1^{\circ} \mathrm{C}\left( \pm 2^{\circ} \mathrm{F}\right)$; and animal carcass and waste storage temperatures below $7^{\circ} \mathrm{C}$. 
- Performance standards describe a desired outcome, provides flexibility in achieving this outcome by granting discretion to those responsible for managing the animal care and use program, the researcher and the Institutional Animal Care and Use Committee (IACUC). The performance approach requires professional input, sound judgment, and a team approach to achieve specific goals (2).

- Practice standard is the application of professional judgment by qualified, experienced individuals to a task or process over time, an approach that has been demonstrated to benefit or enhance animal care and use. Professional judgment comes from information in the peer-reviewed scientific literature, textbooks, etc (2).

In most situations, the Guide is intended to provide flexibility so that institutions can modify practices and procedures with changing conditions and new information (2). AAALAC International recognizes that engineering standards can serve as a useful baseline for some program elements, but considers the application of sound professional judgment to be critical to a successful and contemporary animal care and use program (1).

When interpreting the recommendations of the Guide, it is essential to understand some important terminology. The Guide states that, "Must indicates actions that the Committee for the Update of the Guide considers imperative and mandatory duty or requirement for providing humane animal care and use. Should indicates a strong recommendation for achieving a goal; however, the Committee recognizes that individual circumstances might justify an alternative strategy" (2). The Guide contains a total of 49 imperative and mandatory actions (must) and 684 strong recommendations (should).

\section{AAALAC international perspective and consideration.}

Mandatory item: Given the fundamental importance of the recommendations in the Guide that are prefaced with a "must", AAALAC's CoA typically categorizes site visit findings that do not conform with a "must" statement in the Guide as a mandatory item for correction. In AAALAC's nomenclature, a mandatory item is a serious deviation from the recommendations of the Guide, and/or other AAALAC International standards, which has to be corrected to achieve or continue Full Accreditation. The significance of a mandatory item is ordinarily based on the Council's assessment of its potential to adversely affect the health, well-being or safety of animals or humans (1).

It is also worthwhile to note that several requirements in the Guide are not prefaced with the word "must." Other terminology is occasionally used to convey the same level of importance for complying with the statement (1). Examples include:

- "IACUC is obliged to weigh the objectives of the study against potential animal welfare concerns."

- "Information that is critical to the IACUC's assess- ment of appropriate endpoint consideration in a protocol includes precise definition of the humane endpoint (including assessment criteria), the frequency of animal observation, training of personnel responsible for assessment and recognition of the humane endpoint, and the response required upon reaching the humane endpoint."

- "The committee is responsible for oversight and evaluation of the entire Program and its components...."

- "An integral component of veterinary medical care is prevention or alleviation of pain associated with procedural and surgical protocols."

Suggestions for improvement: The second category of findings identified by AAALAC International site visitors during the on-site assessments of animal care and use programs is comprised of suggestions for improvement (SFIs). These are recommendations that the CoA feels are desirable to upgrade an already acceptable or even commendable program. SFIs are used to draw attention to recommendations that are typically denoted as "should" statements in the Guide. AAALAC considers the offering of SFIs to be an element of the peer review process that is designed to assist accredited programs by sharing the cumulative knowledge and experience of the Council (1).

It should be noted that there is no obligation for institutions to make program changes based on suggestions for improvement; implementation of suggestions is, however, one means of promoting a high quality animal care and use program. Also, a SFI does not automatically become a mandatory item for correction during the next site visit cycle if the same situation is observed. However, if an issue is identified as a SFI but is one of numerous issues noted within the same program area that collectively signal a broader problem, then together they may be determined to be a mandatory item for correction (1).

In summary, while the words "should" and "must" are generally associated with SFIs and mandatory items for correction, respectively, the site visit finding is ultimately judged by the CoA in the context of AAALAC International's Three Primary Standards as well as the scope and impact of the issue (1).

Accreditation process: To become accredited by AAALAC International, an institution must meet all applicable local and national regulations, and the standards outlined in the Guide, Ag Guide, and/or ETS 123 which go beyond the minimums required by law. The standards in the Guide exceed what is required by law. Also, as stated previously, Reference Resources are used by the CoA as applicable (1).

The Council on Accreditation evaluates overall performance and all aspects of an animal care and use program, involving an in-depth, multilayered, peer-review process. Unlike many government regulatory systems, the entire accreditation process is confidential. The accreditation site visit and its results are kept between the organization seeking accreditation and AAALAC International—even if defi- 
ciencies are found. AAALAC International's purpose is to provide a peer-evaluation that results in valuable information organizations can use to improve their programs and achieve new levels of excellence. Members of the Board of Directors, CoA members, ad hoc Consultants/Specialists and AAALAC International office staff are all required to sign confidentiality agreements. Conflict-of-interest statements are also signed by each site visitor to ensure an unbiased review of each program. AAALAC representatives agree to treat all materials as privileged, and safeguard the materials in their possession. Of course, accredited organizations are free to share their AAALAC reports if they choose to do so (1).

\section{Program Description}

AAALAC International evaluates all aspects of an animal care and use program. The Program Description (PD) is the main document used by the CoA to evaluate the animal care and use program of each institution. The PD must be submitted in English with the exception of some appendices (1). Instructions for completing and submitting the PD must be read and followed, http://www.aaalac.org/programdesc/2016_Instructions.pdf/. The process of step-by-step preparation and development of the PD serves as an internal review inherent of each program and to help the institution identify and address weaknesses, resulting in improved animal well-being and better science. The PD includes a description of how a program operates and includes: Section 1, Introduction; Section 2, Description of the animal care and use program management and oversight, animal environment, housing and management, veterinary care, and physical plant; and a variety of appendices.

\section{Document Submission and Fee payment}

The steps in applying for and maintaining AAALAC International accreditation are the same throughout the world. After submission to the AAALAC International office, the PD and application form (in case of the first time new applicant only) undergoes an administrative review to make sure that the program meets the criteria listed in the Rules of Accreditation, http://www.aaalac.org/accreditation/ rules.cfm/. AAALAC International determines the group classification during the review of the application. This classification, based primarily on the size of the facility, and the time necessary to conduct a site visit, establishes the fee schedule for both the one-time application fee and recurring annual fees (1). Organizations seeking accreditation are required to submit a non-refundable application fee following application approval (http://www.aaalac.org/accreditation/fees.cfm/). The application fee covers the cost of the initial site visit. Newly accredited programs also pay a prorated annual fee during the initial year of accreditation. Recurring annual fees cover the cost of regularly scheduled site revisits (1).

\section{Onsite Evaluation (Site Visit)}

In preparation for the site visit, a CoA member who has been assigned to lead the site visit will contact the program's designated point of contact to discuss the scheduling of the site visit. Then, a site-visit team, comprised of two or more site visitors (one CoA member as the team leader and one or more ad hoc specialists depending on the size of the program), is assigned to review the institution's animal care and use program. The site visit team members are experts in animal care and use, as well as in specific areas of research performed at the particular institution. Institutions may request specific expertise be included on the site visit team. It is common that at least one site visitor will have suitable language skills specific to the country and be knowledgeable in the country's specific laws and regulations pertaining to the care and use of animals. Also, it is confirmed that site visitors do not have any conflict of interest with the institution. The AAALAC office provides an official letter that includes the names of the site-visit team members, along with a short biography for each member. In preparation for the site visit, the team will have thoroughly reviewed the PD before arriving.

Then the site visit is conducted. The site visit is not an inspection and it is conducted using a collegial, peer-review approach. The team conducts a thorough document review and a comprehensive facility tour to observe animals and meet with personnel. They collect detailed information in order to prepare a written site visit report for further consideration and deliberation by the CoA. The final outcome of the site visit is determined by the CoA as a result of their review of the report submitted by the site visitors.

The typical site visit schedule will start with an "in-briefing or entrance briefing" during which key institutional members meet with the site visit team to discuss the accreditation process. After the in-briefing, the site visit team will sit down with a smaller group from the institution to go over the PD. The team members may ask specific questions or request additional documents. Next, the team will tour animal facilities. Typically, members of the IACUC are pre-scheduled to meet with the site visit team at some point (usually during lunch) to discuss the committee's activities and other issues pertinent to the animal care and use program. After finishing the tour, the team will review relevant documents and may request additional information on specific protocols or procedures. The site visit team will then meet (alone) to hold an "executive session." During this session, they discuss key observations, review the collective information and identify areas in which the animal care and use program has excelled, and any potential mandatory issues and/or SFIs. Finally, an "exit briefing" is held for all members of the institution that the institution would like to attend. During the briefing, the team shares their key findings and what they intend to recommend to the CoA regarding the accreditation status of the institution. After the visit, the site visitors will document their findings and recommendations in an official site visit report (1). 
The Exit Briefing was adopted as part of the site visit process in 1990 (http://www.aaalac.org/publications/AAALAC i-brief_Exit_Briefing.pdf/) and designed to accomplish several important tasks:

- It provides the institution with the preliminary findings and impressions of the site visit team.

- It provides an opportunity for the institution to correct any misperceptions or provide clarification of the site visit team's preliminary findings.

- It allows the site visit team to explain the "Post Site Visit Communication" (PSVC) process to the institution.

- It is important to note that the exit briefing represents the preliminary collective opinions of the site visitors, which are based on their interpretation of the standards used to evaluate animal care and use programs. These preliminary opinions may or may not reflect the final opinion of the CoA.

\section{After the Site Visit}

The PSVC is a formal letter written by the institution and submitted to AAALAC International within 10 business days after the site visit to explain any immediate, corrective actions that the institution is taking to address the preliminary findings of the site visit team or to correct any misinterpretations made during the exit briefing. The actions in the correspondence will be incorporated to the site visit report and evaluated whether each issue is fully addressed, partially addressed or not addressed at all.

The CoA makes all final decisions regarding accreditation. The CoA meets three times a year (January, May and September) and all site-visit reports are considered during the first CoA meeting that follows the site visit. Prior to the CoA meeting, at least four additional CoA members will review and comment on the report. The CoA member who prepared the report may edit the report to address comments and questions from the reviewers and submit the final report. During the CoA deliberations, the institution's report and a draft letter is considered, and the CoA member who visited the institution will act as the representative of the institution, describing the program and sharing his or her observations. Based on the discussions and deliberations, the accreditation result is determined. After the CoA meeting, the letter is individually reviewed, edited and approved by members of the CoA (Section leader and CoA President) and AAALAC staff. The institution should receive official notification of the results of the accreditation site visit within four to eight weeks after the CoA meeting (1).

\section{CONCLUSIONS}

Categories of accreditation. The CoA evaluates overall performance and aspects of an animal care and use program and institution and determines the accreditation status of individual accreditable units. Results are subject to affirmation by the Board of Directors. The letter explains the commendations and strength of the program, the accredita- tion status, acknowledgement of all actions addressed by the post site visit communication, and all remaining issues, which are categorized as mandatory(ies) and/or SFI(s) as applicable.

A mandatory item is, in Council's judgment, a serious deviation from the recommendations of the Three Primary Standards and/or AAALAC International's Reference Resources, that must be corrected to achieve or continue accreditation. The significance of a mandatory item is ordinarily based on the Council's assessment of its potential to adversely affect the health, well-being or safety of animals or humans.

The possible categories into which a new applicant institution may be placed subsequent to CoA's deliberations are:

- Award Full Accreditation: This is the only category in which the institution is fully accredited.

- Award Accreditation with Condition(s): The institution is accredited but must correct the mandatory item(s) and submit written response actions that addressed all the mandatory issues in the next annual report or within the time period at the discretion of Council provided in the letter.

- Award Provisional Status: The institution is not accredited. The institution must correct the mandatory item(s) and submit written response actions that addressed all the mandatory issues within the time period (up to a total of 24 months from the date of the letter) at the discretion of Council.

- Withhold Accreditation: The institution is not accredited. However, the AAALAC International Bylaws provide the opportunity for the institution to appeal this decision.

After awarded AAALAC International accreditation, the institution will need to submit an "annual report" every year. The report provides current program information, and explains any changes made to animal care and use program during the previous year. To maintain accreditation, subsequent site visits are held every three years and follow the same process described above. The possible categories into which any accredited institution may be placed subsequent to CoA's deliberations are:

- Continued Full Accreditation: This is the only category in which the institution is fully accredited.

- Conditional Accreditation: The institution is accredited but must correct the mandatory item(s) and submit written response actions that addressed all the mandatory issues in the next annual report or within the time period at the discretion of Council provided in the letter.

- Deferred Accreditation: The institution is accredited but must correct the mandatory item(s) and submit written response actions that addressed all the mandatory issues within 2 months from the date of the letter. Failure to fully correct the mandatory issue(s) of Deferred Accreditation will result in Probation.

- Probation: The institution is accredited but must correct the mandatory item(s) and submit written response 
actions that addressed all the mandatory issues within up to a total of 12 months from the date of the letter. If adequate correction of the mandatory item(s) is not achieved within the allotted time, proceedings to revoke accreditation are initiated.

- Revoke Accreditation: As of the date of the letter informing of the Revoke Accreditation status, the institution is no longer accredited. The AAALAC International Bylaws provide the opportunity for the institution to appeal this decision. However, the institution is not accredited during the appeal process. Accreditation is restored by a vote of the Council.

Received February 23, 2017; Accepted March 23, 2017

\section{REFERENCES}

1. AAALAC International. Available from: http://www.aaalac. org/. [accessed 2017 Feb 13].

2. National Research Council (NRC) (2011) Guide for the Care and Use of Laboratory Animals (8th edition), National Academies Press, Washington, DC.

3. Federation of Animal Science Societies (FASS) (2010) Guide for the Care and Use of Agricultural Animals in Research and Teaching ( $3^{\text {rd }}$ edition $)$, FASS, Champlain, IL.

4. Council of Europe (2006) Appendix A of the European Convention for the Protection of Vertebrate Animals Used for Experimental and Other Scientific Purposes (ETS No. 123). Guidelines for Accommodation and Care of Animals (Article 5 of the convention). Approved by the multilateral consultation. Cons, 123, 3. Available from: https://www.aaalac.org/ about/AppA-ETS123.pdf/. [assessed 2017 Feb 13].

5. American Veterinary Medical Association (AVMA) (2013) AVMA Guidelines for the Euthanasia of Animals (2013 edition), AVMA, Schaumburg, IL. Available from: http://avma. org/KB/Policies/Documents/euthanasia.pdf. [assessed 2017 Feb 13].

6. National Research Council (NRC) (1997) Occupational Health and Safety in the Care and Use of Research Animals, National Academy Press, Washington, DC. 